\title{
Shape of the intercondylar notch of the human femur: a comparison of osteoarthritic and non-osteoarthritic bones from a skeletal sample
}

\author{
L Shepstone, J Rogers, J R Kirwan, B W Silverman
}

\begin{abstract}
Objectives-To compare objectively the shape of the intercondylar notch in human osteoarthritic and non-osteoarthritic femora.

Methods-A sample of 96 human femora from a large skeletal population were selected for study. These femora included subjects with evidence of late stage osteoarthritis (that is, with eburnation present) and subjects with no such evidence. The distal end of the femur, viewed axially, was recorded with a video camera, and digitised computer images were produced. The outline of the intercondylar notch was extracted and represented mathematically as two functions. A functional principal components analysis was used to identify important modes of shape variation. These variations in shape were compared between eburnated and noneburnated femora.
\end{abstract}

Results-A statistically significant difference in the shape of the intercondylar notch was found between the two groups. The difference related mostly to the shape of the edge of the medial condyle: in the non-osteoarthritic group this tended to exhibit a concavity; in the osteoarthritic group it tended to be straight.

Conclusions-This observed difference may be a predisposing factor to the development of osteoarthritis. The morphology of the intercondylar notch is related to the functioning of and possible damage to the cruciate ligaments, and damage to the cruciate ligaments is a known risk factor for osteoarthritis. Alternatively, this difference may be due to bony remodelling secondary to the onset of osteoarthritis, perhaps in response to altered biomechanics.

(Ann Rheum Dis 2001;60:968-973)

University of Bristo Division of Medicine, UK

J Rogers

J R Kirwan

Department of

Mathematics,

University of Bristol, UK

B W Silverman

Correspondence to: Dr L Shepstone, School of Health Policy and Practice, University of East Anglia, Norwich NR4 7TJ, UK

L.Shepstone@uea.ac.uk

Accepted 22 March 2001 the knee is damage to the anterior cruciate ligament (ACL) ${ }^{45}$ (indeed, canine models of knee $\mathrm{OA}$ involve the transection of the $\mathrm{ACL}^{6}{ }^{7}$ ), which is essential for the maintenance of mechanical stability of the knee, particularly against excessive external rotation and excessive anterior translation of the tibia on the femur. Any factor which increases the risk of damage to this ligament is, therefore, also a potential risk factor for developing knee OA.

Because the ACL runs through the intercondylar notch of the femur it is reasonable to suppose that the morphology of the notch will influence its functioning and the risk of damage. Numerous studies have examined the relationship between the width of the notch and ACL damage in both humans ${ }^{8-10}$ and animals. ${ }^{11}$

Only one study has, to our knowledge, reported measurements of the intercondylar notch in relation to knee OA. This study by Wada et al examined the width of the notch in relation to the severity of knee OA in cadaveric specimens and also a sample of patients at the time of total knee arthroplasty. ${ }^{12}$ The study found a smaller notch width in those subjects with severe OA than in those with less severe or no OA.

The studies conducted to date assessing the morphology of the intercondylar notch have focused on its width, either as an absolute measure or as a ratio of the notch width to the bicondylar width. A study by Anderson et al, however, also examined the shape of the intercondylar notch using a subjective categorisation. ${ }^{13}$ Five different shape categories were illustrated which, it was suggested, formed a continuum from an "inverted U" shape to a "cresting wave" shape. It was noted that most notches exhibiting narrowing fell towards the wave shaped end of the continuum, though no statistically significant relationship was found. The authors of this study concluded that "the shape of the notch may be of some significance (in relation to ACL damage), though further work is needed in this area." The notch shape may be of importance because femora could be of equal notch width (or notch width index) but have different shapes. Differently shaped notches could provide very different volumes of space for the cruciate ligaments. Alternatively, the shape of the notch may have a more direct impact on the functioning of the ACL.

We carried out a previous study, using human skeletal material, to investigate the shape of the distal femur, comparing osteoarthritic and non-osteoarthritic subjects. ${ }^{14}$ This study suggested that a difference in the shape of the intercondylar notch existed between the two groups. However, this study was not 
directed specifically at intercondylar notch shape and, using a landmark approach to quantify the shape of the distal femur, used little information about notch shape.

Given the potentially important link between the intercondylar notch and knee OA we have carried out a study to compare the notch shape in $\mathrm{OA}$ and non-OA femora using skeletal material. To do this, we quantified intercondylar notch shape in two dimensions, in an objective manner, using all information available.

\section{Material and methods}

The study material comprised a sample of adult femora taken from a large skeletal collection (approximately 2000 adult subjects) excavated from a single site, the churchyard of $\mathrm{St}$ Peter's Church, Barton-on-Humber, in the north of England. This churchyard was in use from around $\mathrm{AD} 900$ until being closed in 1850 . The study sample was originally drawn for an earlier study and has been described elsewhere. ${ }^{14} \mathrm{OA}$ of the knee was defined as the presence of eburnation (indicating fullthickness cartilage loss) anywhere on the distal femur. An attempt was made to identify every subject within the skeletal collection fulfilling this definition, and seek for each, two controls. To qualify as a control, a subject needed to have no evidence of eburnation at any joint site and to have at least one undamaged femur usable in this study. Standard anthropological techniques were used for age and sex determination. ${ }^{15}$ For the purposes of this study, and owing to the limitations of these techniques, subjects were aged as either over or under 45 years at death.

Twenty three subjects were found to have distal eburnation on at least one femur. A pool of potential control subjects was identified. From this pool, 46 subjects were selected to provide as close as possible a group of controls with the same age and sex distribution. Owing to missing femora, this selection resulted in 31 eburnated OA femora and 90 non-eburnated control femora.

Postmortem damage further precluded the use of 25 femora, leaving 96 for study. Seventy two came from 36 subjects with two femora for study and 24 from subjects with only one femur for study. Of the 96 study femora, 21 were in the OA group and 75 in the non-OA control group.

DATA CAPTURE

The distal end of each femur, viewed axially, was filmed with a video camera. The camera was fixed on a horizontal surface. The femora were placed on this surface and allowed to rest naturally on the posterior aspects of the femoral condyles at the distal end and the greater trochanter at the proximal end. This was equivalent to viewing the femur from the distal end with the knee in $90^{\circ}$ of flexion. The distance between the camera lens and each femur was $30 \mathrm{~cm}$. Each femur was rotated in the horizontal plane until the articular surface was parallel with the plane of the camera lens.

Black and white bitmap images $(576 \times 768$ pixels in size) were created by digitising the

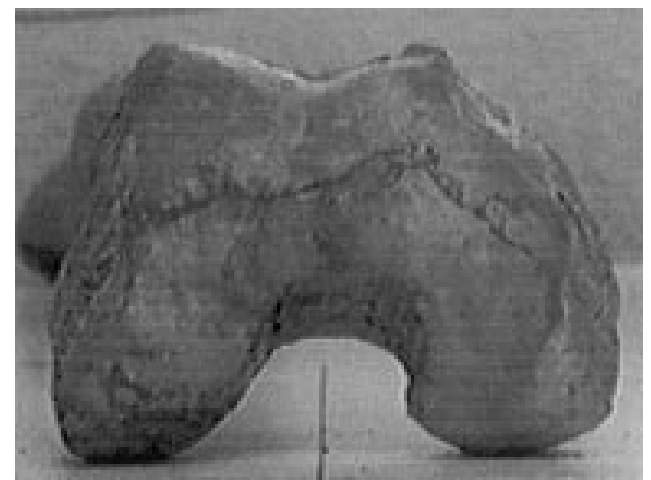

Figure 1 Example of digitised image.

video film. An example is shown in fig 1. All left femora were reflected to produce "right" images, so that the left side of any image indicates the lateral side of the bone and the right side the medial. The outline of the distal femur was extracted by locating those pixels that lay on the border of the bone. This outline was stored as a vector of $\mathrm{x}$ and $\mathrm{y}$ coordinates taken from the border pixel locations.

The region of the intercondylar notch was defined as starting at the most inferior point of the lateral condyle of the border of the bone and continuing medially to the most inferior point of the medial condyle. The most superior point was also noted. Figure 2 indicates these positions. Those points which lay within this region were extracted for analysis. Postmortem damage around the intercondylar notch region of some femora precluded their use.

The length of the intercondylar notch was scaled to be of unit length for each femur. Two functions were used to represent the intercondylar notch, one for the medial edge of the lateral condyle (running from the start of the notch outline to the most superior point) and one for the lateral edge of the medial condyle (running from the end of the notch outline to the most superior point). The functions were translated to remove variation between femora due to horizontal (lateromedial) positioning within bitmaps. The functions were shifted so that the central, vertical axis of each intercondylar notch was zero centred in the lateromedial direction. Further scaling was employed to remove variability between femora due to variable width size. For each femur, the shifted functions were dilated so that the area

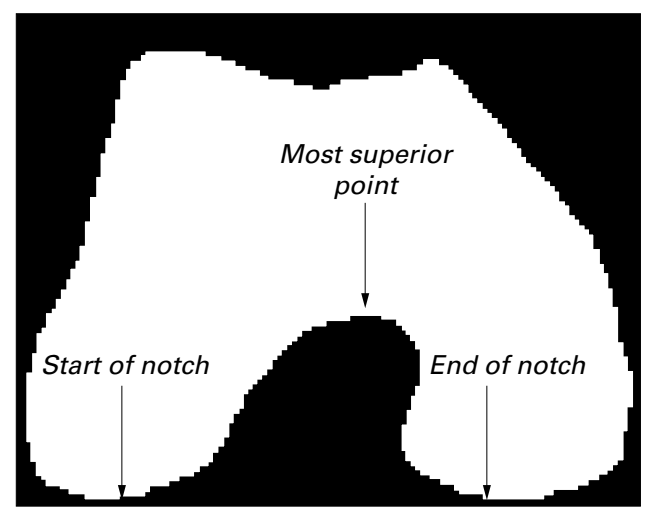

Figure 2 Outline of the intercondylar notch. 
of each intercondylar notch was uniformly adjusted to equal unit area. This process resulted in each intercondylar notch shape being represented as two scaled functions.

STATISTICAL METHODS

A mean intercondylar notch shape was calculated from the individual mean values of each function. Mean shapes were calculated within different subgroups for comparison.

A functional principal components analysis was carried out on the two functions. Computationally, this was achieved using 100 equally spaced points for each function (interpolated using cubic splines), as outlined by Ramsay and Silverman. ${ }^{16}$ Each principal component can be considered as a "mode" of shape variation. ${ }^{17} \mathrm{~A}$ subset of the first principal components was used for further analysis. Each mode of variation captured by these components was illustrated by showing the overall mean shape minus two standard deviations of the component and the overall mean shape plus two standard deviations. Fisher's discriminant function ${ }^{18}$ was used to identify the direction of maximal, between-group shape separation within these components - that is, to identify the mode of intercondylar notch shape variation that best distinguishes different groups of femora. This was also illustrated by showing the overall mean \pm 2 SDs. Generalised estimating equations ${ }^{19}$ with a Gaussian error distribution were used to test formally for betweengroup differences. A canonical correlation analysis ${ }^{18}$ was undertaken to investigate symmetry of the shape of the notch using subjects in which both femora were available for study. Statistical significance was set at the 5\% level.

\section{Results}

Of the 21 femora (seven subjects unilateral and seven bilateral) for which eburnation was evident at the distal end, 15 had eburnation of the lateral patellofemoral region, three of the medial patellofemoral region, two of the medial tibiofemoral region, and one had eburnation of both the medial tibiofemoral and patellofemoral regions. The remaining 75 femora had no evidence of eburnation at the distal end.

Table 1 presents the sex and age distributions within each group. The percentages shown exclude those femora for which information was not available. The eburnated and non-eburnated femora were similar in both age and sex distribution. About $60 \%$ of each group came from women and the majority (around $90 \%$ ) came from subjects aged over 45 at death.

Table 1. Age and sex distributions of 96 femora used for analysis. Data shown as No (\%)

\begin{tabular}{lccc}
\hline & $\begin{array}{l}\text { All femora } \\
(n=96)\end{array}$ & $\begin{array}{c}\text { Eburnated } \\
(n=21)\end{array}$ & $\begin{array}{l}\text { Non-eburnated } \\
(n=75)\end{array}$ \\
\hline Male & $40(43)$ & $8(40)$ & $32(44)$ \\
Female & $53(57)$ & $12(60)$ & $41(56)$ \\
Unsexed & 3 & 1 & 2 \\
& $7(9)$ & $1(11)$ & $6(9)$ \\
$<45$ years & $68(91)$ & $8(89)$ & $60(91)$ \\
$>45$ years & 21 & 12 & 9 \\
Unaged & & & \\
\hline
\end{tabular}

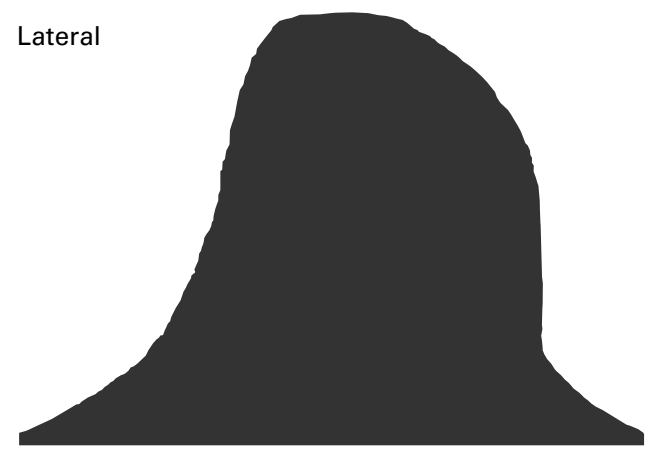

Figure 3 Mean intercondylar notch shape.

Figure 3 shows the mean intercondylar notch shape, using all 96 femora. The lateral aspect of the medial condyle shows a marked concavity. The medial aspect of the lateral condyle is, in comparison, much flatter. The notch appears to turn laterally in the superior direction.

The first five principal components accounted for over $81 \%$ of the total sample variation. The sixth component accounted individually for less than $3 \%$ of the total variation. The first five components were, therefore, used for further analyses.

Figures $4 \mathrm{~A}-\mathrm{E}$ illustrate the modes of shape variation captured by these components (mean \pm 2 SDs). The first component illustrates variability mainly with respect to the lateral aspect of the medial condyle. Interestingly, this component seems to reflect the continuum of shapes illustrated by Anderson et al; the negative end corresponds to the wave shaped end of their spectrum and the positive to the inverted $U$ shaped end. The second captures variability, mainly in the shape of the medial aspect of the lateral condyle. The third and fourth components capture complex variability in the shape of both condyles, contrasting straight edges with more curved edges. The fifth component captures relatively minor variability, mainly in the medial aspect of the lateral condyle.

Figures $5 \mathrm{~A}$ and $\mathrm{B}$ show the mean eburnated and non-eburnated intercondylar notch shapes. Figure 6 illustrates the mode of shape variation best discriminating between these two groups. There was a significant mean difference in this mode of shape variation between eburnated and non-eburnated femora ( $p=0.002$, generalised estimating equation). The eburnated femora appear, on average, to have straighter, less concave medial edges than the non-eburnated femora. Further, the eburnated femora had generally more symmetrical, straight shapes compared with the laterally curving shapes of the non-eburnated femora.

The same process was carried out to compare shape differences between age groups and sexes. There was no significant difference between age groups ( $p=0.152$, generalised estimating equation), but there was a significant difference with respect to sex $(p=0.022$, generalised estimating equation). Figure 7 shows the mean shapes and fig 8 illustrates the mode of shape variation best discriminating between the sexes. The male intercondylar 
A

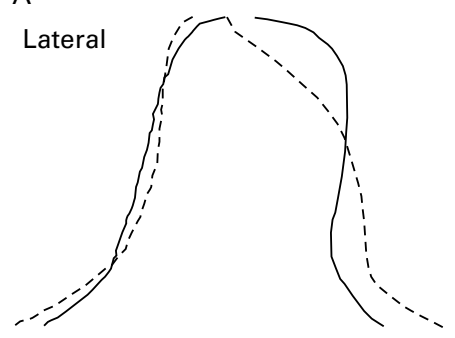

First component (34\%)
B

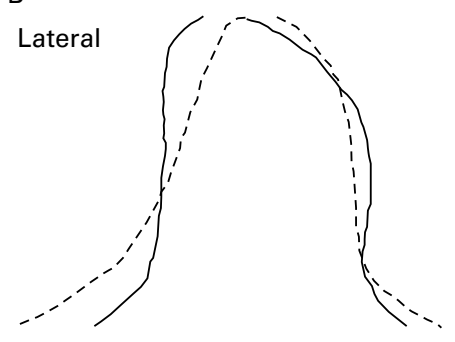

Second component (24\%)
C

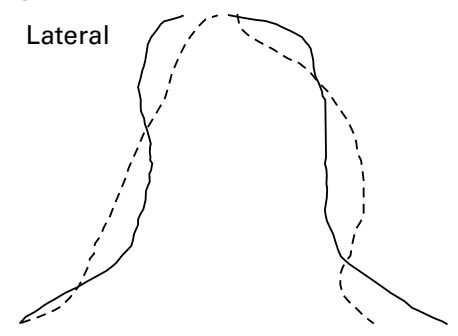

Third component (10\%)
D

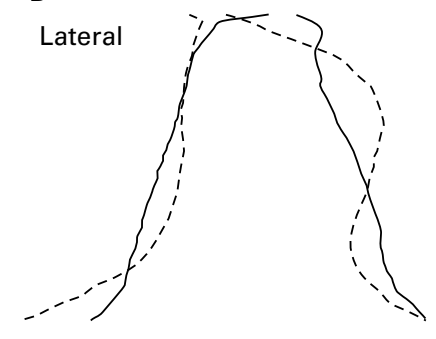

Fourth component (8\%)
E

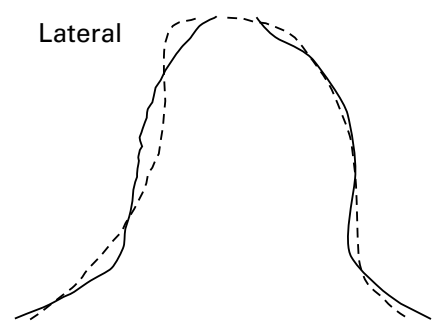

Fifth component (5\%)

Figure 4 Illustration of first five principal components (with percentage of total variation). Solid line $=$ mean $+2 S D$ s of the principal component; dashed line $=$ mean $-2 S D$ s of the principal component .

notches (figure 7A) appear, on average, to have a greater concavity of the medial edge and a lateral "turn" in the superior direction. The difference between men and women is similar
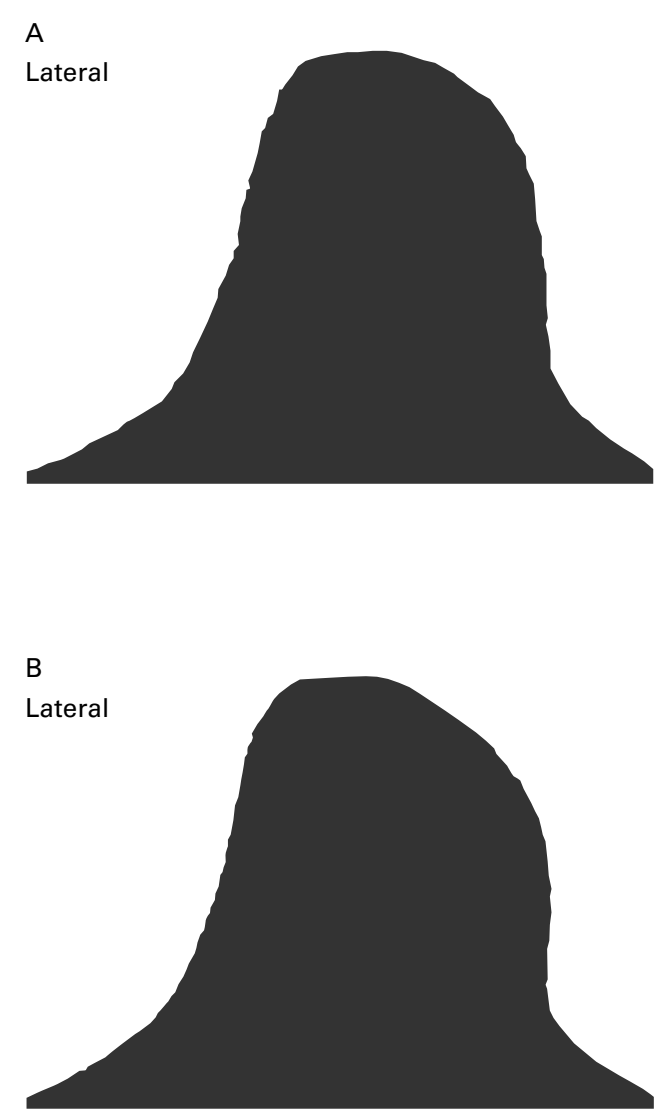

Figure 5 (A) Eburnated and (B) non-eburnated mean intercondylar notch shapes. to that between the non-eburnated and eburnated femora.

The canonical correlation analysis was carried out on the 72 femora from the 36 subjects with both left and right femora present for study in order to investigate the possibility of symmetry in the shape of the intercondylar notch. The first four canonical correlation coefficients were all statistically significant and ranged in value from 0.826 to 0.529 , providing evidence of symmetry of the left and right intercondylar notch shapes.

\section{Discussion}

It has frequently been suggested, ${ }^{8-10}$ with evidence, that the width of the intercondylar notch of the femur is related to the occurrence of ACL damage: smaller notches are more likely to be associated with ACL damage than larger ones. It has further been suggested that the shape of the notch may also be important. Given a relationship between cruciate ligament

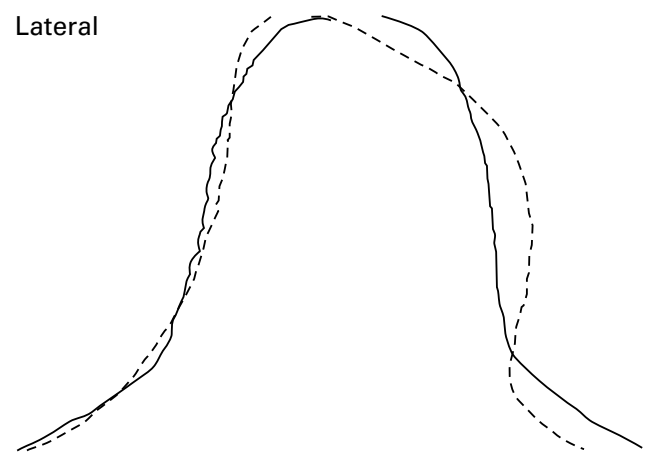

Figure 6 Illustration of discriminant function between eburnated and non-eburnated femora. Solid line $=$ mean + $2 S D$ s; dashed line $=$ mean $-2 S D$ s. 
A

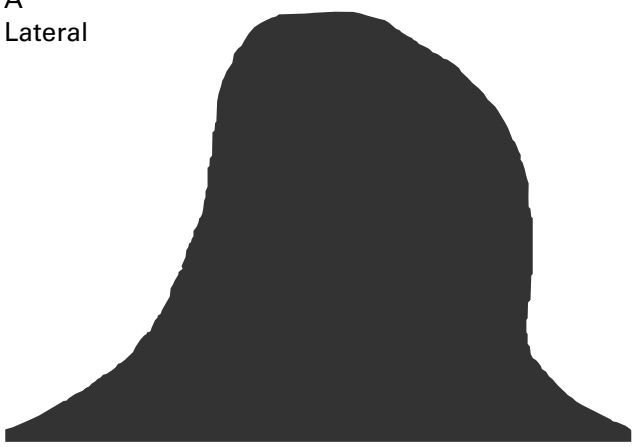

B

Lateral

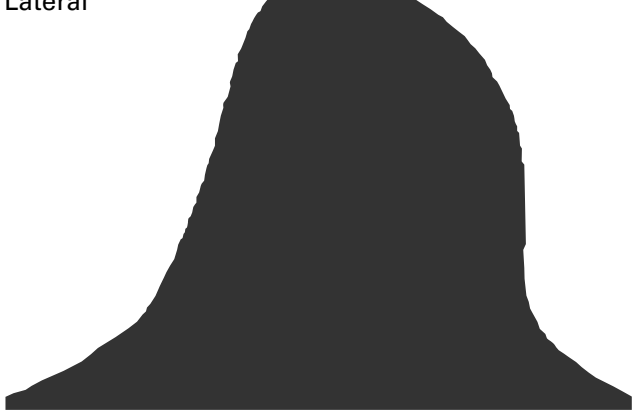

Figure 7 (A) Male and (B) female mean intercondylar notch shapes.

damage and knee OA it is a logical step to consider the relationship between intercondylar notch morphology and knee OA directly.

Skeletal material lends itself well to the study of $\mathrm{OA}^{20}$ because patent signs of its presence can be viewed clearly and directly. However, it has clear limitations. Any study using skeletal material is necessarily cross sectional and cannot involve any clinical features of knee OA. The definition of OA for this study was based upon the presence of eburnation of the articular surface. This is indicative of full thickness cartilage loss and therefore late stage OA. Possibly, subjects in our control group also had $\mathrm{OA}$, but at an earlier stage. The effect of this misclassification would be an underestimation of the true difference in notch shape between $\mathrm{OA}$ and non-OA femora.

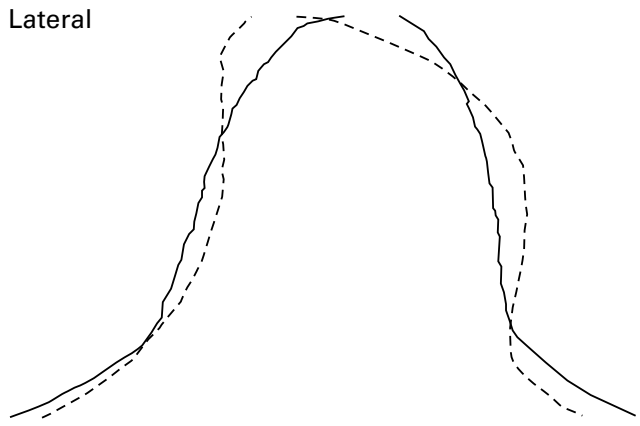

Figure 8 Illustration of discriminant function between men and women. Solid line $=$ mean $+2 S D s ;$ dashed line $=$ mean-2SDs
Functional principal components analysis, combined with classical discriminant analysis, provided a means of quantifying shape variability and assessing between-group differences while facilitating a visual presentation of those differences and overall variability. We found considerable variability in the shapes of the intercondylar notch of the femora we examined. The first principal component appeared to capture the spectrum of shapes suggested by Anderson $e t a l,{ }^{13}$ and accounted for one third of the sample variation. It should be noted that this degree of variation is pertinent to this study sample which, given the age and sex distribution, is not representative of the general population; possibly, in the general population this spectrum may account for a different proportion of variation and perhaps be a more "important" spectrum of shapes. The remaining components illustrated further modes of complex shape variability.

We found a statistically significant difference in the shape of the intercondylar notch between the OA and non-OA control groups. This difference is concordant with our previous study, ${ }^{14}$ but because many of the femora used here were also used in the previous study it would be inappropriate to consider this as a confirmatory study. Rather, it provides a more detailed and informative analysis of intercondylar notch shape. The difference was largely in the lateral aspect of the medial condyle, with the non-OA group exhibiting, on average, a wave shaped notch, whereas the OA group exhibited a more inverted $U$ shaped notch. Interestingly, Anderson et al, reported that the wave shaped notch related to a smaller width, which is perhaps contradictory to what was observed here. One might naturally expect the OA group to have more narrow notches and thus have the wave shaped notch. However, in the study by Anderson et al there was no statistically significant relationship, and classification of notch shape was a subjective matter.

The observed difference in shape might be congenital and one that increases the risk of ACL damage (or perhaps alters knee mechanics through some other route) and is, therefore, a genuine risk factor for knee OA. However, other than a possible decrease in the overall size of the notch, the effect of the observed shape difference on the ACL is not immediately apparent. The medial aspect of the lateral condyle is known to impinge on the ACL in hyperextension or external rotation of the tibia, but little difference was found for this side of the notch. An alternative explanation of the shape difference is simply that it is due to a remodelling of the medial condyle (consistent with a varus deformity) and the development of osteophytes purely secondary to the onset of OA. This may be a phenomenon related neither to the initial development nor the subsequent progression of knee OA. A third possibility is that the shape difference is indeed secondary to the onset of $\mathrm{OA}$, but the remodelling of the intercondylar notch then leads to ACL damage, which in turn leads to joint instability (or further joint instability), resulting in an 
acceleration of the condition. This might explain why some patients with knee OA may remain relatively stable over a period of years, whereas others may deteriorate rapidly in a short space of time. This scenario is essentially the hypothesis of Wada et al. ${ }^{12}$ In their study of notch width and knee OA they reported that damage to the ACL was only found in those subjects with severe disease and suggested that a narrowing of the notch occurs after the onset of OA, leading to ACL damage, knee instability, and disease progression.

We found no evidence of a difference with respect to age group. This is perhaps not surprising, however, because very few subjects included in the study sample were estimated to be under the age of 45 years at death.

We found a statistically significant difference with respect to sex. This again included not only a difference in the lateral aspect of the medial condyle (men having generally a more concave edge) but also included a marked difference in the medial aspect of the lateral condyle and also the superior lateral corner of the notch. This difference is unlikely to be an effect of the OA difference because the sex distribution of the $\mathrm{OA}$ and non-OA groups was very balanced. A difference in the medial aspect of the lateral condyle was also noted. It is of interest to note that some studies have reported a difference in notch width between men and women. Shelbourne et al found that women have smaller notches than men, irrespective of height. ${ }^{10}$ Souryal et al also found that the intercondylar notch width index was smaller in women than in men and stated that this was not due to a disparity in bone size or overall size but simply that the notch in men actually occupies relatively more space in the distal femur than in women. ${ }^{9}$ This is not a universal finding, however, as neither LaPrade and Burnett nor Teitz et al found a statistically significant difference in notch width between men and women. ${ }^{2122}$ The issue, in the context of OA, is an important one. The risk of knee $\mathrm{OA}$ is higher in women than men. ${ }^{23}$ One would, therefore, expect risk factors for knee OA also to vary between the sexes, of which this might be an example.

This study has identified a systematic difference in the shape of the intercondylar notch of the femur between eburnated and noneburnated femora. We have taken the presence of eburnation as indicative of late stage OA. Possibly, a difference in the shape of the intercondylar notch is a risk factor for knee OA but this can only be speculated about given the cross sectional nature of the study. Further clinical studies would be necessary to provide conclusive evidence.

We thank Gerry Barber for her assistance with this project. This work comprises part of the doctoral work by Dr L Shepstone, supported by a generous studentship (K0516) from the Arthritis Research Campaign.

1 van Saase JLCM, van Romunde LKJ, Cats A, Vandenbroucke JP, Valkenburg HA. Epidemiology of osteoarthritis: Zoetermeer survey. Comparison of radiological osteoarthritis in a Dutch population with that in 10 other populations. Ann Rheum Dis 1989;48:271-80.

2 Spector TD, Hart DJ. How serious is knee osteoarthritis? Ann Rheum Dis 1992;53:565-8.

3 Dieppe P. Osteoarthritis: are we asking the wrong questions? Br J Rheumatol 1984;23:161-5.

4 Kannus P, Järvinen M. Posttraumatic anterior cruciate ligament insufficiency as a cause of osteoarthritis in a knee joint. Clin Rheumatol 1989;8:251-60.

5 Jacobsen K. Osteoarthrosis following insufficiency of the cruciate ligaments in man: a clinical study. Acta Orthop Scand 1977;48:520-6.

6 Pond MJ, Nuki G. Experimentally-induced osteoarthritis in the dog. Ann Rheum Dis 1973;32:387-8.

7 Brandt KD, Braunstein EM, Visco DM, O'Connor B, Heck Brandt KD, Braunstein EM, Visco DM, O'Connor B, Heck
$\mathrm{D}$, Albrecht $M$. Anterior (cranial) cruciate ligament transection in the dog: a bone fide model of osteoarthritis, not merely of cartilage injury and repair. J Rheumatol 1991;18:436-46.

8 Good L, Odensten M, Gillquist J. Intercondylar notch measurements with special reference to anterior cruciate ligament surgery. Clin Orthop 1991;263:185-9.

9 Souryal TO, Freeman TR. Intercondylar notch size and anterior cruciate ligament injuries in athletes: a prospective study. Am J Sports Med 1993;21:535-9.

10 Shelbourne KD, Davis JT, Klootwyk TE. The relationship between intercondylar notch width of the femur and the incidence of anterior cruciate ligament tears: a prospective study. Am J Sports Med 1998;26:402-8.

11 Aiken SW, Kass PH, Toombs JP. Intercondylar notch width in dogs with and without cranial cruciate ligament injuries. in dogs with and without cranial cruciate ligament injuries. ogy 1995;8:128-32.

12 Wada M, Tatsuo H, Baba H, Asamoto K, Nojyo Y. Femoral ntercondylar notch measurements in osteoarthritic knees. Rheumatology (Oxford) 1999;38:554-8.

13 Anderson AF, Lipscomb AB, Liudahl KJ, Addlestone RB. Analysis of the intercondylar notch by computed tomography. Am J Sports Med 1987;15:547-52.

14 Shepstone L, Rogers J, Kirwan JR, Silverman, B. The shape of the distal femur: a comparison between eburnated and non-eburnated. Ann Rheum Dis 1999;58:72-8.

15 Brothwell D. Digging up bones. London: British Museum of Natural History, 1981.

6 Ramsay JO, Silverman BW. Functional data analysis. New York: Springer, 1997.

17 Cootes TF, Taylor CJ, Cooper DH, Graham J. Training models of shape from sets and examples. Proceedings of British machine vision conference. Leeds: Springer, 1992:918.

18 Gnanadesikan R. Methods for statistical data analysis of multivariate observation. 2nd ed. Chichester: Wiley, 1997.

19 Liang KY, Zeger SL. Longitudinal data analysis using generalized linear models. Biometrika 1986;73:13-22.

20 Jurmain RD, Kilgore L. Skeletal evidence of OA: a palaeopathological perspective. Ann Rheum Dis 1995;54: 443-50.

21 LaPrade RF, Burnett QM. Femoral intercondylar notch stenosis correlation to anterior cruciate ligament injuries. Am J Sports Med 1994;22:198-203.

22 Teitz CC, Lind BK, Sacks BM. Symmetry of the femoral notch width index. Am J Sports Med 1997;25:687-90.

23 Felson DT, Zhang Y, Hannan MT, Naimark A, Weissman B, Aliabadi P, et al. Risk factors for incident radiographic knee osteoarthritis in the elderly: the Framingham study. Arthritis Rheum 1997;40:728-33. 